THE ANALYST.

\title{
THE EFFECT OF LIGHT UPON SOME REAGENTS AND CHEMICAL COMPOUNDS.
}

By T. P. BLunt, M.A. Oxon., F.C.S.

Read before the Society of Public Analysts, on 17th March, 1880.

THE following remarks are for the most part of a practical character, and intended to point out the importance to the chemist of a consideration of the effects of exposure to light upon some of the ordinary reagents of the laboratory, which have not been usually regarded as subject to its influence. My attention was first drawn in this direction in the course of an investigation into the action of light on certain organisms, upon which Dr. A. H. Downes and I have been engaged for more than two years. Nearly all our results have been embodied in a series of papers read before the Royal Society, but it may not be considered impertinent to reproduce here such of them as have a bearing upon the practical question of the preservation of reagents. I do not pretend that all the facts detailed below are new, but some of them have certainly not received the attention they deserve. Oxalic acid forms a most useful basis for a standard solution, owing to the ease 
with which it is obtained in a pure state by crystallization, and subsequently weighed, but it has fallen into disrepute on account of the instability of the normal solutions prepared with it; now it has been clearly proved by us that this instability is solely due to the action of light, and that normal volumetric oxalic solution (6.3 per cent.) may be preserved unchanged for any period in the dark. The same remark applies even to the decinormal $(0.63$ per cent.) solution, if the precaution be taken of first boiling to destroy germs or organisms, otherwise it may become turbid and lose strength in the dark. In the light decinormal oxalic solution is rapidly destroyed by oxidation. In one of our experiments, made in test tubes, partially filled, and plugged with cotton wool, six months' insolation, between Jan. 21st and June 15th, sufficed to destroy both acid taste and reaction, apparently leaving nothing behind but pure water-the carbonic acid formed having of course escaped. I have already, in the columns of The ANaLyst, called attention to the fact that a weak permanganate solution, such as that used by Tidy in water analysis, is perfectly permanent for one month when kept in the dark. My experience extends now over a longer period, and I find that such a solution may be preserved unchanged in the dark for many months, in spite of variations of temperature. Potassium iodide and ferrous iodide are a pair of compounds whose conduct under light is mutually anomalous ; solid potassium iodide in presence of light and moisture soon becomes tinged with yellow through the liberation of iodine, while it is a fact well known to pharmacists that in order to preserve the syrup of iodide of iron (ferrous iodide) from discolouration it should be placed in white glass bottles in the strongest available light. We once half filled a test tube with the syrup and exposed it in a window, in the summer. Each morning a brown layer appeared on its surface, the accumulation of the past night. This gradually disappeared in the sunlight, so that before evening the contents of the tube had once more become completely colourless, and this phenomenon occurred as long as the experiment was under observation. It was satisfactorily proved that the only effect of the sugar was to render the solution more stable, for aqueous solutions of ferrous iodode were found to become discoloured much more rapidly in the dark than when exposed to sunlight. Solutions of potassium iodide behave in an exactly opposite manner to those of ferrous iodide in all the respects mentioned above; they become discoloured under sunlight, but can be preserved indefinitely in the dark, and if a dilute solution which has turned yellow in the light be afterwards screened from it, the colour very gradually disappears.

The cause of the discolouration of potassium iodide by light having been much discussed of late years, it may perhaps be worth while to give a somewhat detailed account of our own attempts at the solution of the problem. Four tubes containing a weak solution of the salt, which filled them to about ore-third of their capacity, were exhausted at the sprengel pump; air which had been freed from carbonic and all other acids by long contact and frequent agitation with caustic potash was then admitted by a special arrangement, and the tubes were sealed off; two were insolated and two encased. At the same time some more of the solution was placed in four ordinary test tubes, which were simply plugged with cotton wool, and exposed to sunlight and darkened respectively in a similar manner to the previous pairs. All the insolated tubes rapidly became coloured, no difference being noticeable between the rates of colouration in the tubes containing ordinary and purified air, those kept in the dark being perfectly preserved. A pair of tubes was now charged with solution, exhausted and sealed off. No change took place on insolation, and thus the 
somewhat improbable alternative of mere dissociation was disposed of, and the conclusion remained that the effect of light was due to direct oxidation, without the intervention of any acid.

There can be little doubt that solution of potassium iodide of any strength could be perfectly preserved in a well-stoppered bottle in the dark, and would be much more convenient than the fragments of solid salt we are most of us in the habit of using. A similar reaction to that observed in the case of potassium iodide under light occurrs with dilute solutions of all the alkaline oxalates, which are gradually oxidized, with the formation of carbonates, but much less rapidly than a solution of oxalic acid of corresponding strength. In operating on solutions containing the same proportion of acid radical it was found that the decomposition was nearly equal in the case of the oxalates of sodium, potassium, and lithium, but somewhat greater in that of the ammonium salt; hence it would appear to be desirable to keep solutions of ammonium oxalate in the dark when they are required to be preserved of uniform strength-as for instance, where they are employed in the volumetric estimation of lime.

In the course of one of our experiments two tubes were partially filled over mercury with a mixture of atmospheric air and ammoniacal gas, one was then exposed to light and the other darkened. On examining the tubes after many months the mercury had risen in the encased tube. The surface of the metal was blackened and its convexity lost, theso appearances being evidently due to oxidation. In the tube in the light, on the other hand, the level of the mercury remained unchanged, and its surface was clean, bright and convex.

These results afford strong presumptive evidence against the formation of ozone in sunlight, and consequently against the views of those writers who have ascribed some of the phenomena of oxidation under light to its interrention, for it is well known that a trace of ozone in air is capable of destroying the convexity and lustro of the mercurial surface.

Dr. Dupré said he heard a similar paper read some time ago, and he went the next day and examined his decinormal oxalic acid which had been made about 14 months, and standing in a place never struck by sunlight. He had some of the exact acid with which it was made and could certainly find no difference between the two.

Dr. Muter said that had it been in the light it might have been affected. He thought it was probably through germs if any change took place. If no germs could get in it a solution might remain correct for a number of years.

Mr. Hehner pointed out that some germs were not fond of light as they flourished much better in the dark. If fungus were exposed to the sunlight it would very quickly die.

Dr. Bartlett said that he had some potassium iodide which had been for about $\mathbf{2 0}$ years in the dark in his laboratory, and it had become of a deep yellow colour. 